\title{
Intrinsic Spin-Orbit Coupling in Zigzag and Armchair Graphene Nanoribbons
}

\author{
Ying Li, ${ }^{1}$ Erhu Zhang, ${ }^{1}$ Baihua Gong, ${ }^{1}$ and Shengli Zhang, \\ ${ }^{1}$ Department of Applied Physics, Xi'an Jiaotong University, Xi'an 710049, China \\ ${ }^{2}$ MOE Key Laboratory for Nonequilibrium Synthesis and Modulation of Condensed Matter, Xi'an Jiaotong University, \\ Xi'an 710049, China
}

Correspondence should be addressed to Shengli Zhang, zhangsl@mail.xjtu.edu.cn

Received 26 December 2010; Accepted 23 February 2011

Academic Editor: Shaogang Hao

Copyright (C) 2011 Ying Li et al. This is an open access article distributed under the Creative Commons Attribution License, which permits unrestricted use, distribution, and reproduction in any medium, provided the original work is properly cited.

\begin{abstract}
Starting from a tight-binding model, we derive the energy gaps induced by intrinsic spin-orbit (ISO) coupling in the lowenergy band structures of graphene nanoribbons. The armchair graphene nanoribbons may be either semiconducting or metallic, depending on their widths in the absence of ISO interactions. For the metallic ones, the gaps induced by ISO coupling decrease with increasing ribbon widths. For the ISO interactions, we find that zigzag graphene nanoribbons with odd chains still have no band gaps while those with even chains have gaps with a monotonic decreasing dependence on the widths. First-principles calculations have also been carried out, verifying the results of the tight-binding approximation. Our paper reveals that the ISO interaction of graphene nanoribbons is governed by their geometrical parameters.
\end{abstract}

\section{Introduction}

Graphene, first obtained in 2004 [1], has been explored for spintronics due to its unique and intriguing properties [2]. In spintronics, one of the main interactions that could affect the spin behavior is intrinsic spin-orbit (ISO) coupling. In the last few years, a volume of research has been performed on the ISO coupling in graphene [3-8] and shows that the ISO coupling can cause a gap in the band structure. Recently, it is reported that the geometric curvature $[5,9,10]$ and impurity [11] can enhance the effective strength of the ISO by orders of magnitude.

When graphene is patterned into a narrow ribbon, one obtains a graphene nanoribbon (GNR), whose carriers are confined to the one-dimensional (1D) system. There are two basic shapes of GNRs, called armchair GNRs (AGNRs) and zigzag GNRs (ZGNRs). Both results by tight-binding(TB) method [12-15] and first-principles calculations [16-19] show that spectra of GNRs depend strongly on the shapes of their edges. GNRs, offering a system to investigate confinement effects, could be key elements in future electronics and spin-transport applications. In the case of GNRs with ISO coupling, on one hand, based on TB model, Zarea et al.
[20-22] have studied the effects of ISO interactions on lowenergy properties of AGNRs and ZGNRs. However, these works were based on the effective $\pi$-orbital TB approaches and used the model from graphene plane by imposing hardwall boundary conditions. They did not study ISO from that of each atom. They also did not give the exact data of the gap induced by ISO coupling. On the other hand, based on first principles, few investigations of the ISO interaction on GNRs have so far been performed. Thus, the ISO coupling is still significant and challenging in GNR.

In this paper, we study the ISO effects on ZGNRs and AGNRs by the $s p^{3}$ TB method from a graphene ribbon model. The first-principles calculations are also performed to verify the results. Our investigation reveals how the geometrical parameters, such as width and the edge shape, affect the strength of the ISO.

\section{Tight-Binding Model}

Two basic kinds of GNRs are shown in Figure 1. The AGNRs are classified by number of dimer lines while ZGNRs by number of chains. To simplify, we use $N$-AGNR and $N$-ZGNR to represent the AGNR with $N$ dimer lines and 


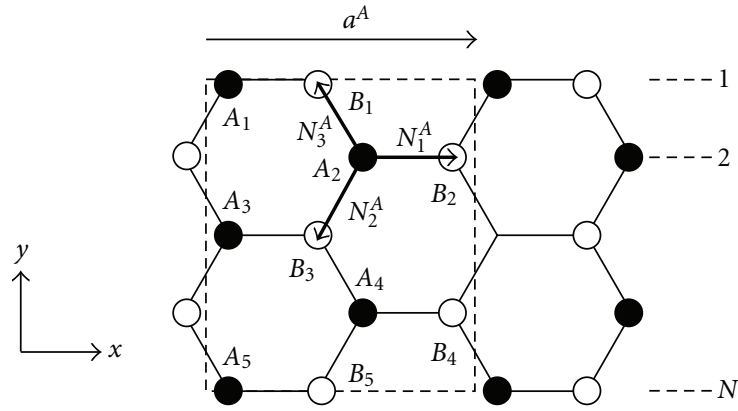

(a)

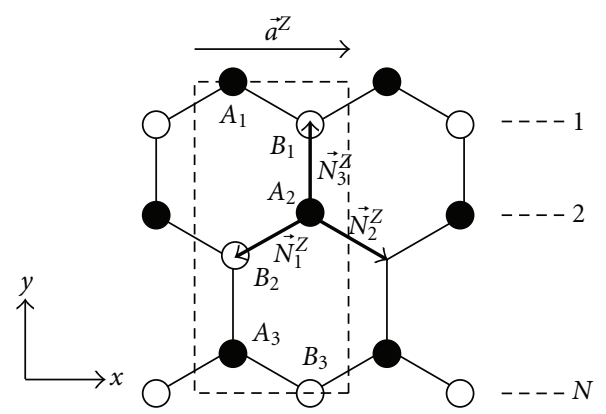

(b)

Figure 1: The geometry of (a) N-AGNR and (b) N-ZGNR.

the ZGNR with $N$ chains, respectively. As seen in Figure 1, a GNR is described by a rectangular unit cell containing $2 N$ carbon atoms. The $2 N$ atoms can be classified to $N$ subsystems. Each subsystem contains two atoms $A$ and $B$. The unit vector $\vec{a}^{A}\left(\vec{a}^{Z}\right)$ denotes the translational axis of an (a) AGNR (ZGNR). The nearest-neighbor translation vectors of the atoms are

$$
\begin{aligned}
\vec{N}^{A} & =\left\{\vec{N}_{1}^{A}, \vec{N}_{2}^{A}, \vec{N}_{3}^{A}\right\} \\
& =\left\{a\left(\frac{1}{\sqrt{3}}, 0\right), a\left(-\frac{1}{2 \sqrt{3}},-\frac{1}{2}\right), a\left(-\frac{1}{2 \sqrt{3}}, \frac{1}{2}\right)\right\}, \\
\vec{N}^{Z} & =\left\{\vec{N}_{1}^{Z}, \vec{N}_{2}^{Z}, \vec{N}_{3}^{Z}\right\} \\
& =\left\{a\left(-\frac{1}{2},-\frac{1}{2 \sqrt{3}}\right), a\left(\frac{1}{2},-\frac{1}{2 \sqrt{3}}\right), a\left(0, \frac{1}{\sqrt{3}}\right)\right\},
\end{aligned}
$$

where $a=2.46 \AA$. We employ the tight-binding method within a two-center Slater-Koster approximation for nearestneighbor hopping, and orthogonality between Wannier functions centered on different atoms is assumed. This gives the elements of the Hamiltonian matrix [4]

$$
\begin{aligned}
& h_{A, \mu ; A, \mu^{\prime}}(\vec{k})=h_{B, \mu ; B, \mu^{\prime}}(\vec{k})=t_{\mu} \delta_{\mu, \mu^{\prime}}, \\
& h_{A, \mu ; B, \mu^{\prime}}(\vec{k})=h_{B, \mu^{\prime} ; A, \mu}^{*}(\vec{k})=\sum_{i=\text { nearest }} e^{i \vec{k} \cdot \vec{N}_{i}} t_{\mu, \mu^{\prime}}\left(\vec{N}_{i}\right),
\end{aligned}
$$

where $\mu\left(\mu^{\prime}\right)=s, p_{x}, p_{y}, p_{z}, A$ and $B$ represent the two distinct sites in the honeycomb lattice unit cell. $t_{\mu}$ is the atomic energies of $\mu$ orbital, and $t_{\mu, \mu^{\prime}}$ denotes the hopping energies between the nearest-neighbor sites. In Table 1, we reproduce for completeness the relationship between $t_{\mu, \mu^{\prime}}$ and the four independent Slater-Koster parameters. To simplify, we let $\theta=(s s \sigma), \alpha=(s p \sigma), \beta=(p p \sigma)$, and $\eta=(p p \pi)$. The wave vector $k$ is parallel to $x$.

First, we consider the $\pi$ electrons. The $2 \times 2$ hamiltonian matrix $H_{\pi \mathrm{mn}}$ is introduced to describe the interaction of
TABLE 1: Two-center matrix elements for hopping between $s$ and $p$ orbital along a direction specified by the unit vector $\left(n_{x}, n_{y}, n_{z}\right)$, taken from [4]. $s=-8.868 \mathrm{eV}, p=0,(s s \sigma)=-6.769 \mathrm{eV},(s p \sigma)=$ $5.580 \mathrm{eV},(p p \sigma)=5.037 \mathrm{eV}$, and $(p p \pi)=-3.033 \mathrm{eV}$.

\begin{tabular}{llll}
\hline$t_{s}$ & $s$ & $t_{p_{x}, p_{x}}$ & $n_{x}^{2}(p p \sigma)+\left(1-n_{x}^{2}\right)(p p \pi)$ \\
$t_{p}$ & $p$ & $t_{p_{y}, p_{y}}$ & $n_{y}^{2}(p p \sigma)+\left(1-n_{y}^{2}\right)(p p \pi)$ \\
$t_{s, s}$ & $(s s \sigma)$ & $t_{p_{z}, p_{z}}$ & $n_{z}^{2}(p p \sigma)+\left(1-n_{z}^{2}\right)(p p \pi)$ \\
$t_{s, p_{x}}$ & $n_{x}(s p \sigma)$ & $t_{p_{x}, p_{y}}$ & $n_{x} n_{y}(p p \sigma)-n_{x} n_{y}(p p \pi)$ \\
$t_{s, p_{y}}$ & $n_{y}(s p \sigma)$ & $t_{p_{x}, p_{z}}$ & $n_{x} n_{z}(p p \sigma)-n_{x} n_{z}(p p \pi)$ \\
$t_{s, p_{z}}$ & $n_{z}(s p \sigma)$ & $t_{p_{y}, p_{z}}$ & $n_{y} n_{z}(p p \sigma)-n_{y} n_{z}(p p \pi)$ \\
\hline
\end{tabular}

$m$ and $n$ subsystems. The $2 N \times 2 N$ hamiltonian matrix for $\mathrm{N}$-GNR can be written as follows:

$$
\begin{aligned}
& H_{\pi} \\
& =\left(\begin{array}{ccccccc}
H_{\pi 1,1} & H_{\pi 1,2} & 0 & \cdots & 0 & 0 & 0 \\
H_{\pi 2,1} & H_{\pi 2,2} & H_{\pi 2,3} & \cdots & 0 & 0 & 0 \\
\vdots & \vdots & \vdots & & \vdots & \vdots & \vdots \\
0 & 0 & 0 & \cdots & H_{\mathcal{A}, N-2} & H_{\mathcal{A}, N-1} & H_{\mathcal{A}, N} \\
0 & 0 & 0 & \cdots & 0 & H_{\pi N, N-1} & H_{\pi N, N}
\end{array}\right),
\end{aligned}
$$

where $\mathcal{A}$ denotes $\pi N-1$.

We can diagonalize the above hamiltonian and gain the eigenvalues for GNRs. The results are the same with those of [12]. The energy spectra of AGRNs for different widths can be observed in Figure 2. The system is metallic for AGNRs with widths $N=3 M-1$, where $M$ is an integer, and insulating otherwise. For the metallic AGNRs, the top of valence band and the bottom of conduction band are degenerate at $K^{A}=0$ in the Brillouin zone (BZ). For the insulating ones, the gaps decrease with an increase in ribbon widths and tend to zero in very large $N$. The calculated band structures of ZGNRs are depicted in Figure 3 for four different ribbon widths. It is observed that for ZGNRs, the valence band and the conduction band are degenerate at $K^{Z}=\pi / a$ and $K^{Z^{\prime}}=-\pi / a$ in the $\mathrm{BZ}$. 


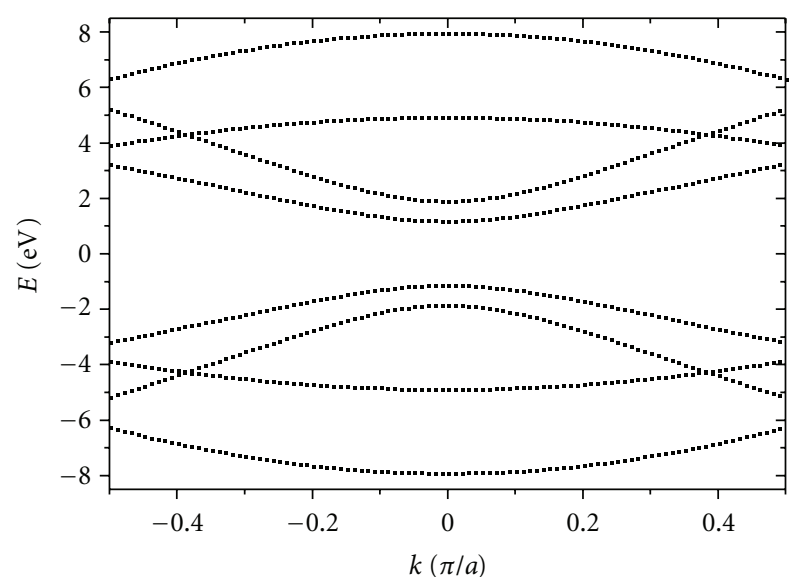

(a)

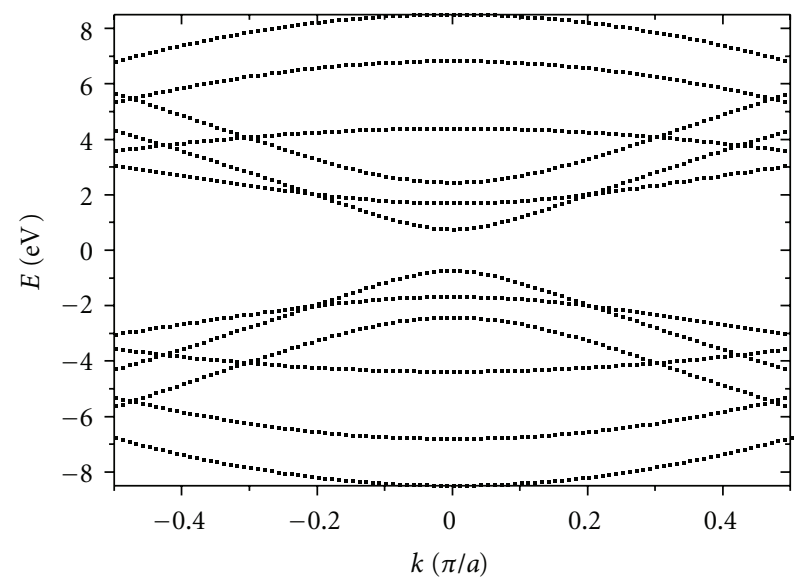

(c)

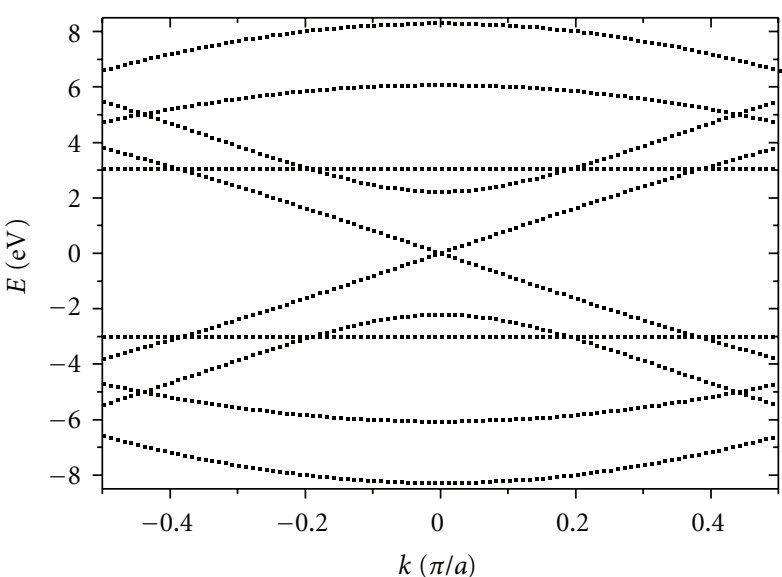

(b)

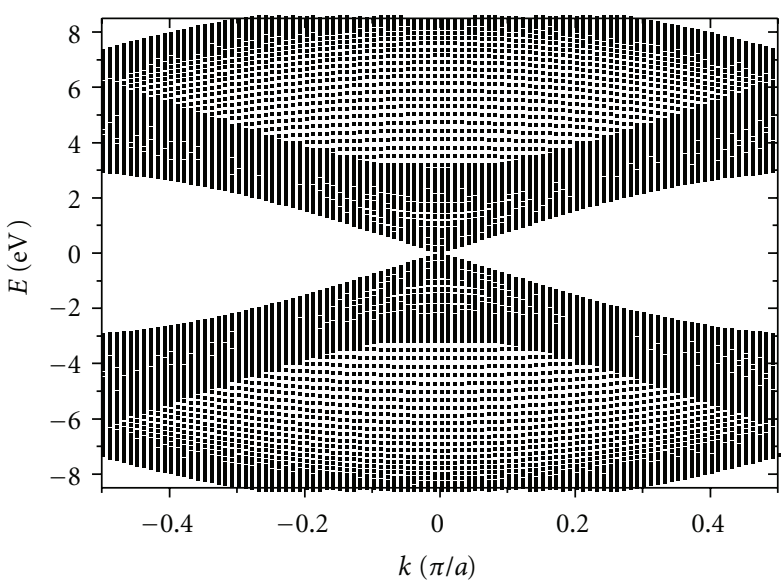

(d)

Figure 2: Calculated band structures of AGNRs with (a) $N=4$, (b) $N=5$, (c) $N=6$, and (d) $N=60$.

\section{Intrinsic Spin-Orbit Coupling}

$H_{\mathrm{SO}}$ induces $\sigma-\pi$ coupling. So, we discuss the hamiltonian including both $\pi$ and $\sigma$ electrons. The hamiltonian for $N$-GNR is an $8 N \times 8 N$ matrix:

$$
\begin{aligned}
& H_{\pi \sigma} \\
& =\left(\begin{array}{ccccccc}
H_{1,1} & H_{1,2} & 0 & \cdots & 0 & 0 & 0 \\
H_{2,1} & H_{2,2} & H_{2,3} & \cdots & 0 & 0 & 0 \\
\vdots & \vdots & \vdots & & \vdots & \vdots & \vdots \\
0 & 0 & 0 & \cdots & H_{N-1, N-2} & H_{N-1, N-1} & H_{N-1, N} \\
0 & 0 & 0 & \cdots & 0 & H_{N, N-1} & H_{N, N}
\end{array}\right) .
\end{aligned}
$$

We consider the ISO interaction as an additional term to $H_{\pi \sigma}$. The ISO interaction $H_{\mathrm{SO}}$ of the system comes from each atomic ISO hamiltonian $h_{\mathrm{SO}} . h_{\mathrm{SO}}$ is in the form of $h_{\mathrm{SO}}=\xi \vec{L}$. $\vec{S}$, where $\xi=6 \mathrm{meV}$ denotes the ISO coupling constant. $\vec{L}$ and $\vec{S}$ represent the angular momentum operator and the spin operator, respectively. The hamiltonian $h_{\mathrm{SO}}$ can be expressed as follows [3-5]:

$$
h_{\mathrm{SO}}=\left(\begin{array}{cccccccc}
0 & 0 & 0 & 0 & 0 & 0 & 0 & 0 \\
0 & 0 & \frac{i \xi}{2} & 0 & 0 & 0 & 0 & \frac{\xi}{2} \\
0 & -\frac{i \xi}{2} & 0 & 0 & 0 & 0 & 0 & \frac{i \xi}{2} \\
0 & 0 & 0 & 0 & 0 & \frac{\xi}{2} & \frac{i \xi}{2} & 0 \\
0 & 0 & 0 & 0 & 0 & 0 & 0 & 0 \\
0 & 0 & 0 & \frac{\xi}{2} & 0 & 0 & -\frac{i \xi}{2} & 0 \\
0 & 0 & 0 & -\frac{i \xi}{2} & 0 & \frac{i \xi}{2} & 0 & 0 \\
0 & \frac{\xi}{2} & -\frac{i \xi}{2} & 0 & 0 & 0 & 0 & 0
\end{array}\right) \text {, }
$$

which acts on the basis states $|s \uparrow\rangle,\left|p_{x} \uparrow\right\rangle,\left|p_{y} \uparrow\right\rangle,\left|p_{z} \uparrow\right\rangle$, $|s \downarrow\rangle,\left|p_{x} \downarrow\right\rangle,\left|p_{y} \downarrow\right\rangle$, and $\left|p_{z} \downarrow\right\rangle$. Then, the total hamiltonian including ISO interaction is $H=H_{\pi \sigma}+H_{\text {SO }}$. Finally, we solve the total hamiltonian for GNRs and obtain the eigenvalues. 


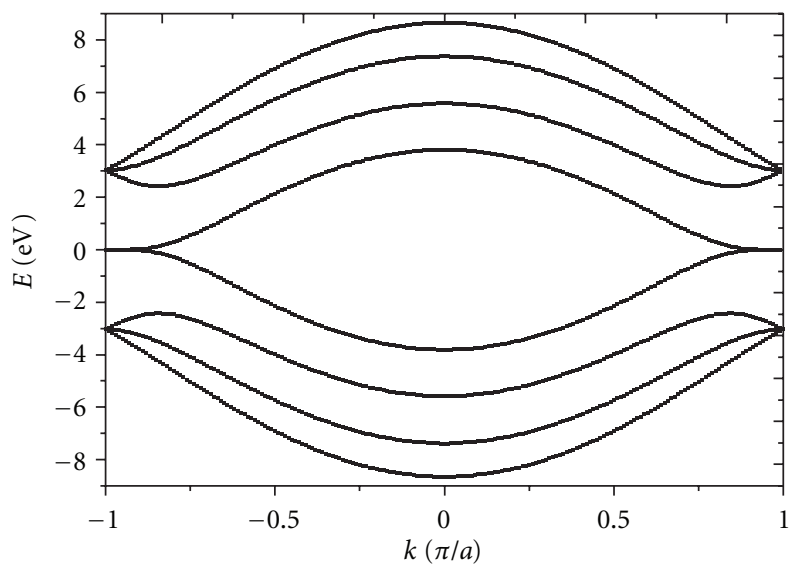

(a)

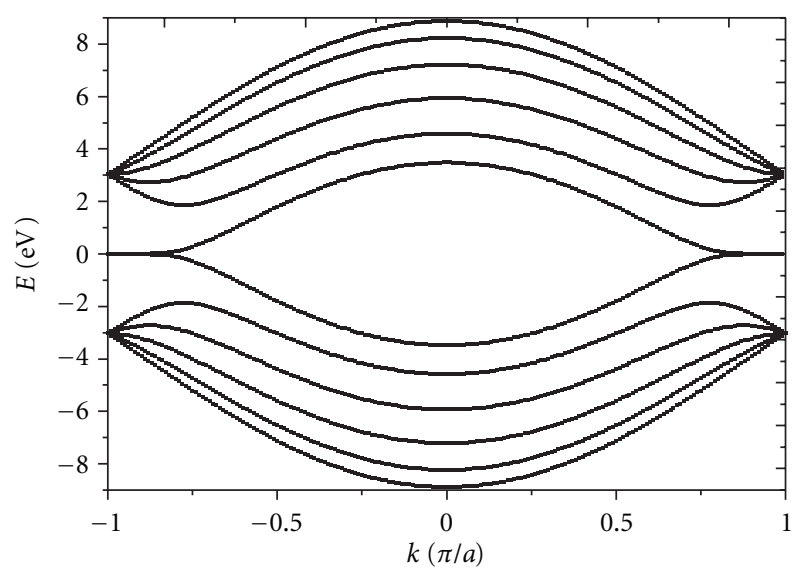

(c)

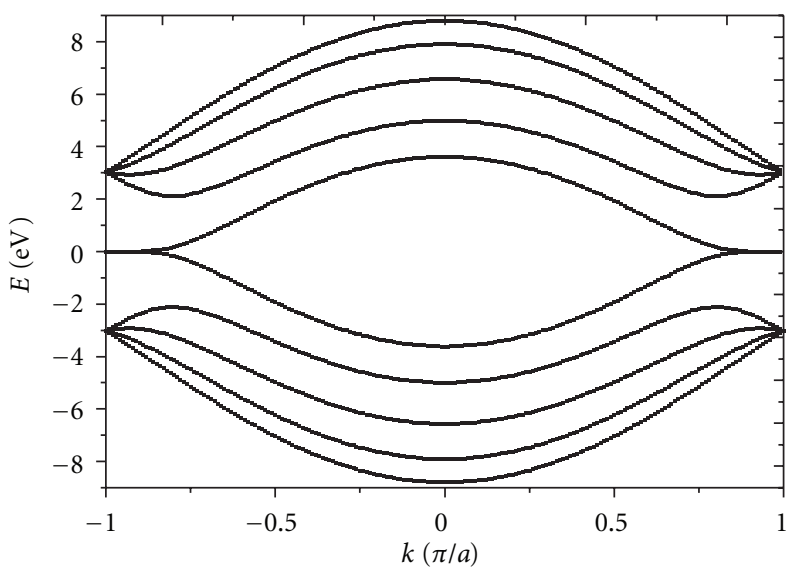

(b)

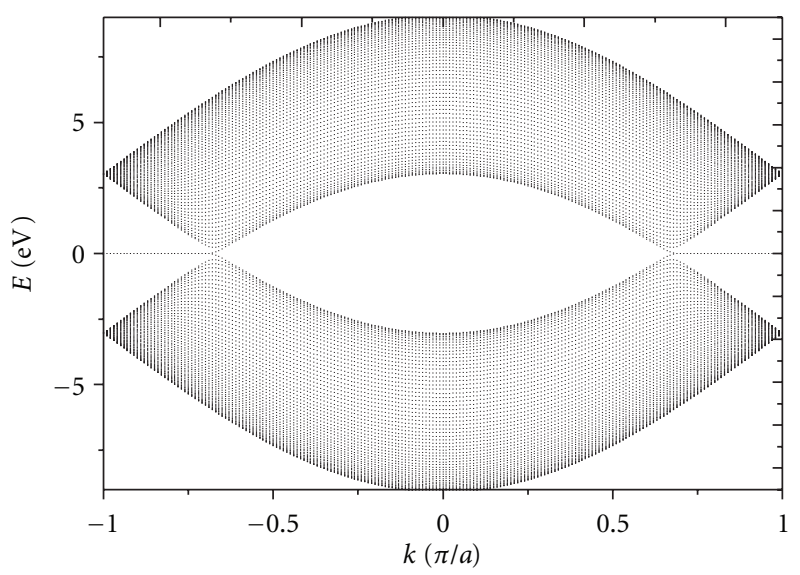

(d)

Figure 3: Calculated band structures of ZGNRs with (a) $N=4$, (b) $N=5$, (c) $N=6$, and (d) $N=60$.

Since it has been shown that the ISO coupling in graphene just causes a small gap [3-8], thus the spectra near the Fermi surface attract our interest. Hence, we focus on the metallic GNRs. If ISO interaction is taken into account, the band structures are lifted. In metallic AGNRs and the ZGNRs with even chains, a gap is opened in the spectra by ISO coupling. In contrast, for the ZGNRs with odd chains, conduction and valence edge bands are still degenerated at $K^{Z}=\pi / a$. The difference between the influence on odd ZGNRs and even ZGNRs is likely due to their configuration. The odd ZGNRs are in "zigzag/antizigzag" configuration while even ZGNRs are in "zigzag/zigzag" configuration. We display the energy spectrum near Fermi surface of 5-AGNR with ISO interaction near $K^{A}$ point in Figure 4(a). The band gap caused at $k=0$ is $1.7 \times 10^{-3} \mathrm{meV}$. The band structures of 5-ZGNR and 4-ZGNR with ISO interaction near $K^{Z}$ point are presented in Figures 4(b) and 4(c). For 4-ZGNR, the highest valence band state and the lowest conduction band state are located at $k=0.9973 \pi / a$ rather than $k=\pi / a$ point. There is a band gap of $1 \times 10^{-5} \mathrm{meV}$ induced by ISO. The ISO interaction is shown to have a stronger effect in 5-AGNR than in 4-ZGNR. It is possible because that the proportion of edge atoms in unit cell atoms which is $4 / 10$ for 5-AGNR is larger than $2 / 8$ for 4 -ZGNR. The relation between the gaps and the widths will be discussed in the next section to compare the results with the first-principles calculations data.

\section{First-Principles Calculations}

In order to verify the results from TB model, we study the metallic GNRs by first-principles calculations. The relativistic $a b$ initio electronic structure calculations for graphene nanoribbons have been performed using the Vienna ab initio simulation package (VASP) [23]. We choose the projector augmented wave (PAW) method [24] with a Perdew-BurkeErnzerhof (PBE) generalized gradient exchange correlation potential (GGA) to describe the electron interactions [25]. A vacuum separation of $10 \AA$ is used to eliminate interactions between graphene layers. An energy cutoff of $950 \mathrm{eV}$ and Monkhorst-Pack k-point meshes are employed to deal with the many atoms in a unit cell of the GNRs for various widths. The ISO interaction is calculated considering only the spherical part of the potential inside muffin tins surrounding the carbon nuclei. The edge carbon atoms of our GNRs are 


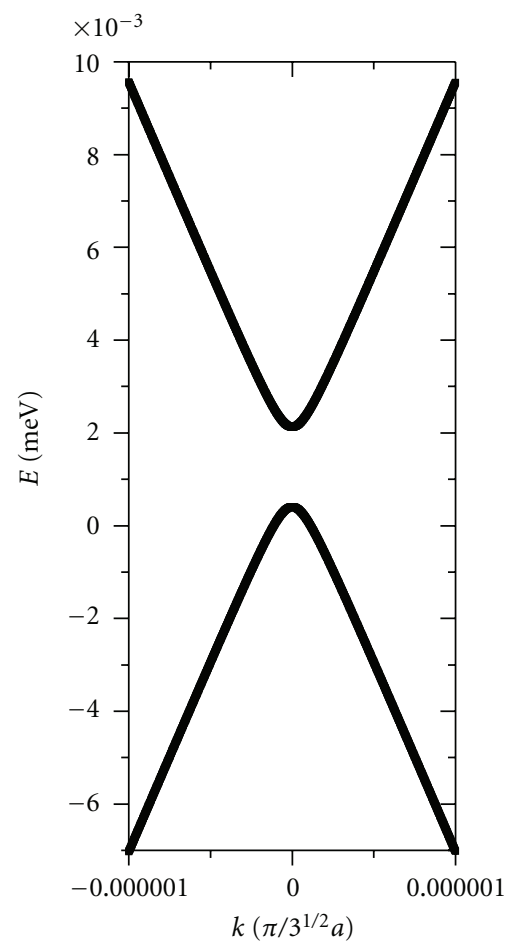

(a)

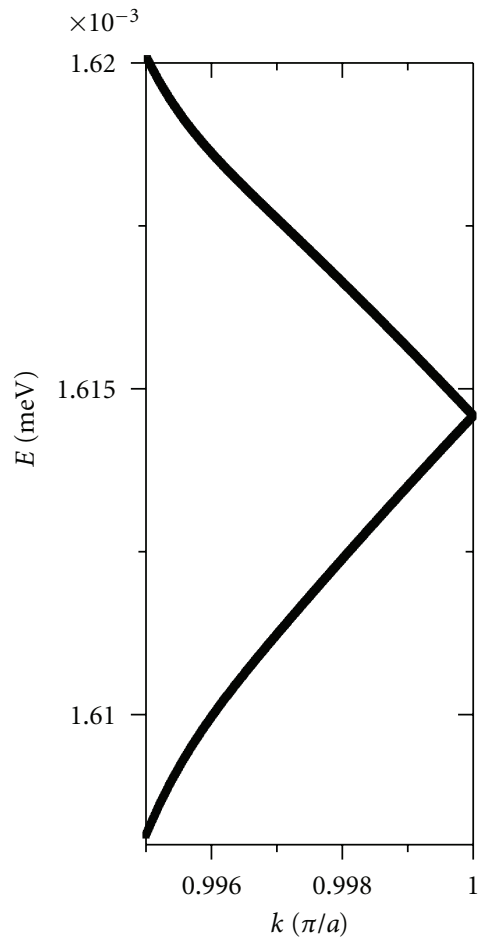

(b)

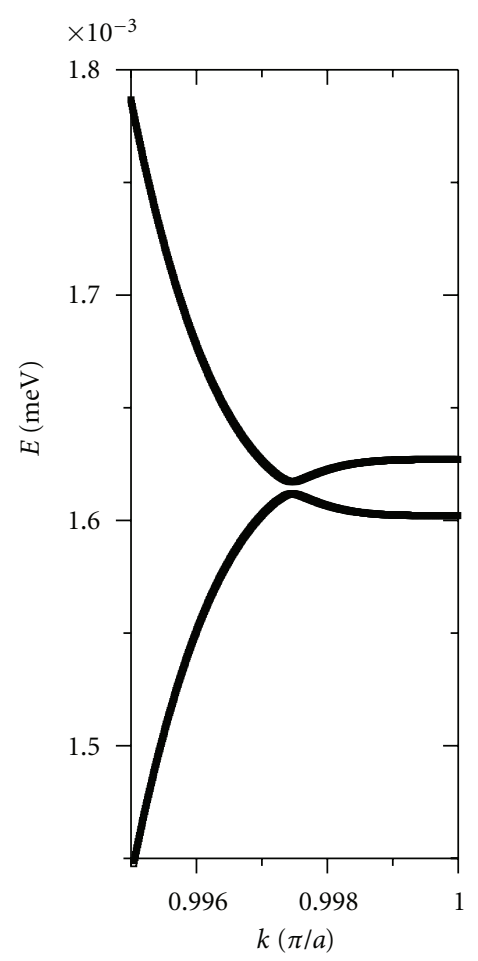

(c)

FIGURE 4: The band structures of the GNRs with ISO interaction for (a) 5-AGNR, (b) 5-ZGNR, and (c) 4-ZGNR. The band gaps are $1.7 \times 10^{-3} \mathrm{meV}, 0$, and $1 \times 10^{-5} \mathrm{meV}$, respectively.

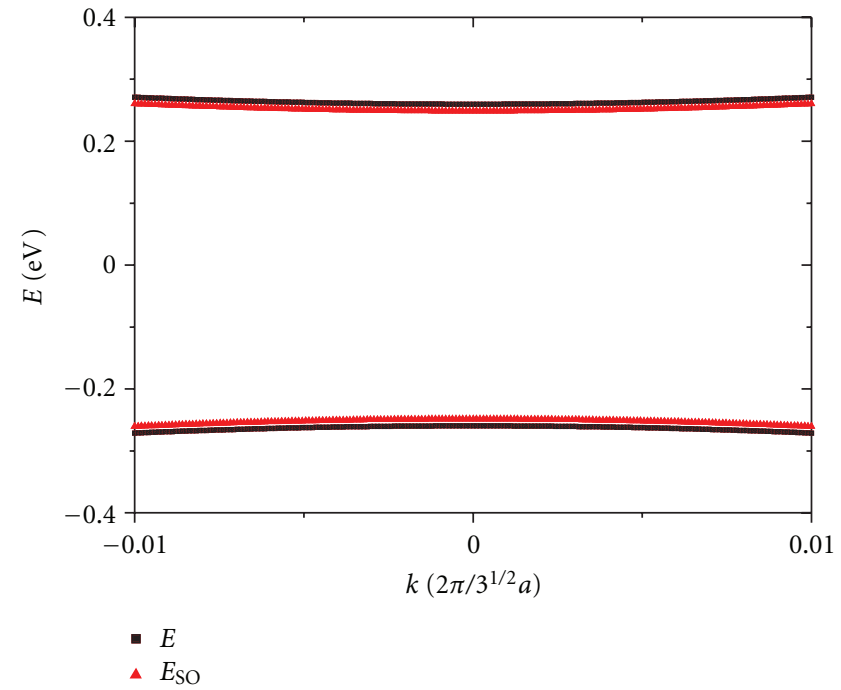

FIGURE 5: (Color online) Band structure of the 5-AGNR without (black squares) and with (red triangles) ISO coupling obtained by first-principles calculations.

passivated by hydrogen atoms. Due to the smallness of ISOderived gaps in GNRs, we artificially increase the relativistic potential in VASP code in our calculations.

First, we study the band structures of AGNRs with different widths $N=3 M-1$ in the range of $N=5$ to $N=35$.

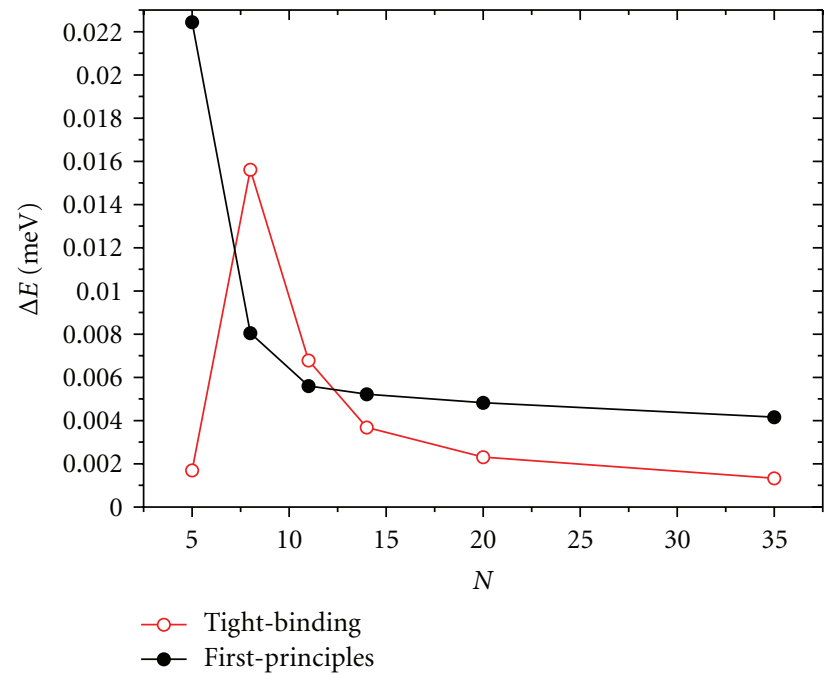

Figure 6: (Color online) The relationship between the gaps induced by ISO and N-AGNRs from tight-binding approximation (red circles) and from First-principles calculations (black diamonds; energies are multiplied by 50 ).

We have known that the AGNRs with $N=3 M-1$ show the metallic behaviors in TB method without ISO interactions, and gaps are opened by the ISO coupling between the valence band and the conduction band. While for the first principles calculations, we find that there are always gaps in the band 


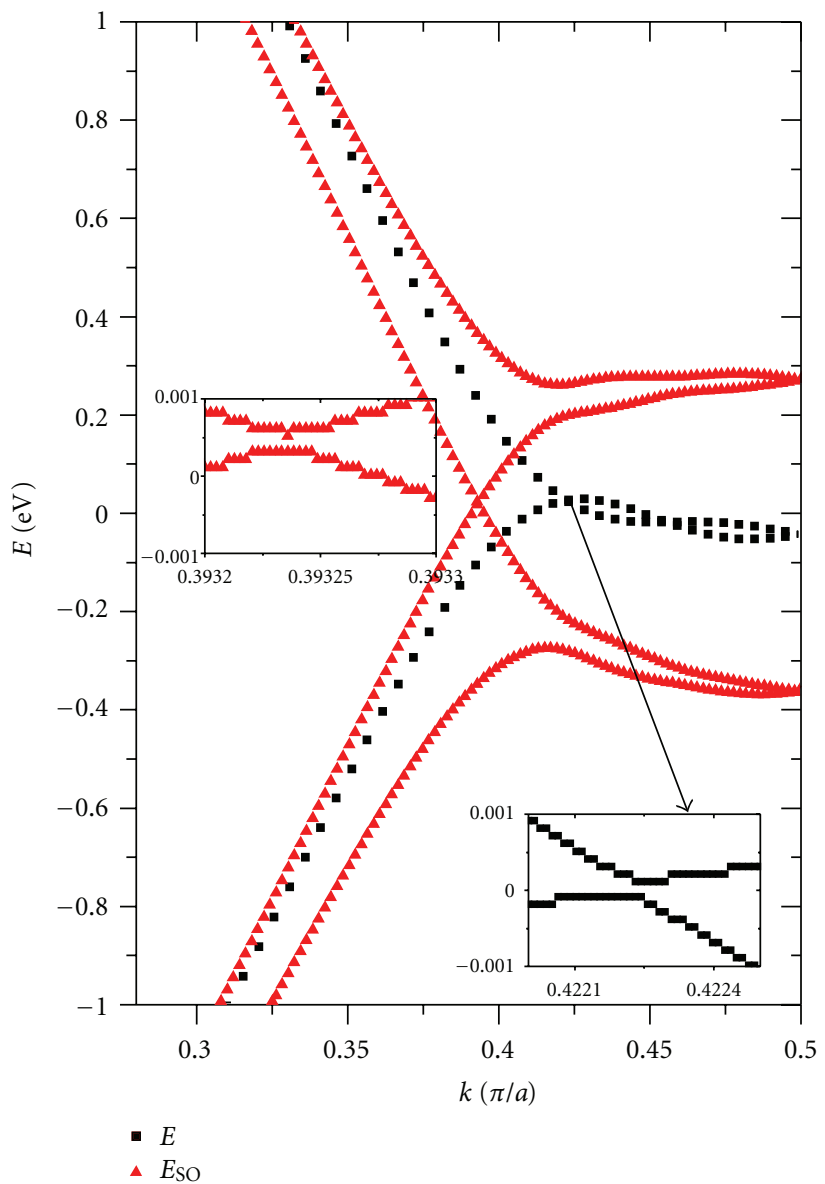

(a)

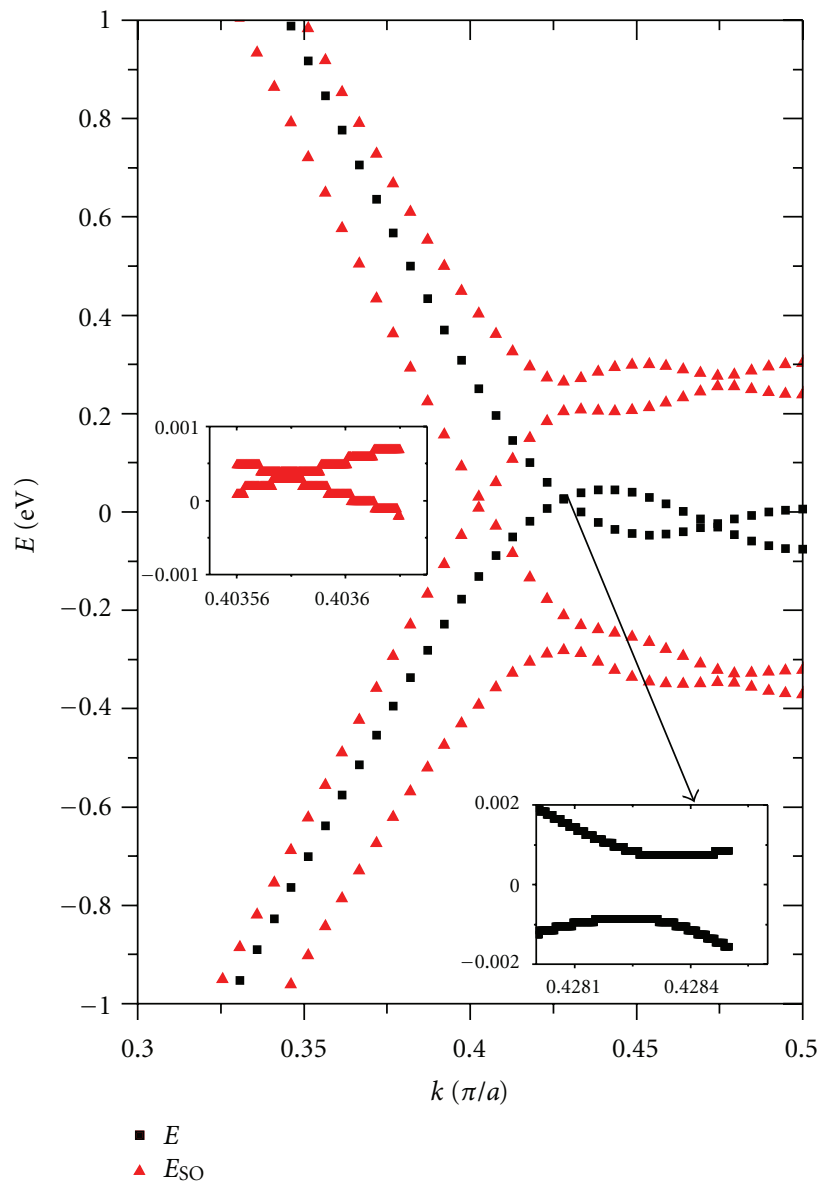

(b)

FIGURE 7: (Color online) Band structures of the (a) 5-ZGNR and (b) 4-ZGNR without (black squares) and with (red triangles) ISO coupling obtained by first-principles calculations. The insets are a zoom in of the band spectra close to the Fermi surface.

spectra, but the ISO coupling can reduce the gaps. The firstprinciples band structure near Fermi surface of 5-AGNR is shown in Figure 5. Without ISO interaction, the band gap is $0.5379 \mathrm{eV}$. When the ISO coupling is considered, the band gap becomes smaller. As Figure 6 depicts, the change of the gap $\Delta E$ decreases when $N$ increases from 8 to 35 , which is in good agreement with the results of TB method except $N=5$. In 5-AGNR, the gap change is smaller in TB method than that expected. In TB method, we do not consider the change of the carbon-carbon bond length on the edge. As the smallest width metallic AGNR, 5-AGNR, is affected most by the edge. So, the model may not exactly express the ISO interaction in 5-AGNR.

Next, we consider the ZGNRs with the widths for $N=$ $3,4,5,6$. Without ISO coupling, although the bands are self-avoiding, the ZGNRs are metallic. In the presence of ISO interaction, the bands split, and a gap is opened. Figure 7 displays the band structures near Fermi surface of 5-ZGNR and 4-ZGNR in the presence and absence of the ISO interaction. Increasing the relativistic potential by 300 times, the band gap changes from $1.9 \times 10^{-3} \mathrm{eV}$ to $1.0 \times$ $10^{-5} \mathrm{eV}$. Since the ISO-opened gap is the second power of the potential expected from the perturbation theory, final results which are obtained through dividing the gaps gotten from VASP by 90000 are $2.0 \times 10^{-6} \mathrm{meV}$ and $1.0 \times 10^{-7} \mathrm{meV}$, respectively. This method was verified of being effectual by the first-principles calculations in [4]. Both first-principles calculations and $\mathrm{TB}$ approximation demonstrate that with the increase of the widths for even chains, the gaps decrease rapidly.

\section{Summary}

In summary, we have investigated the ISO effects in ZGNRs and AGNRs within four orbital TB approximation and firstprinciples calculations. In TB method, for metallic AGNRs and the ZGNRs with even chains, a gap is opened in the spectra by ISO coupling while for the ZGNRs with odd chains, conduction and valence edge bands are still degenerated at $K^{Z}=\pi / a$. By first-principles calculations, the ISO coupling reduces the gaps for AGNRs with $N=$ $3 M-1$ and causes a gap in ZGNRs. The results show that the edge configuration and width affect the strength of the ISO coupling. The ISO interaction has a stronger effect 
for AGNRs than for ZGNRs. Besides, the influence of ISO coupling is weaker on GNRs with larger width, which the edge affects less.

Note Added. Recently, a related preprint which addresses spin-orbit interactions in GNRs with more than 50 chains by four orbital TB method has been posted [26].

\section{Acknowledgments}

The authors thank X. H. Zhang, Q. Wang, M. G. Xia, D. Q. Liu, N. Ma, and H. W. Chen for valuable discussions. This paper is supported by the Cultivation Fund of the Key Scientific and Technical Innovation Project, Chinese Ministry of Education (no. 708082) and NSFC with Grant 11074196. One of the authors (E. Zhang) is also supported by the Fundamental Research Funds for the Central Universities (no. xjj20100088).

\section{References}

[1] K. S. Novoselov, A. K. Geim, S. V. Morozo et al., "Electric field effect in atomically thin Carbon films," Science, vol. 306, pp. 666-669, 2004.

[2] A. H. Castro Neto, F. Guinea, N. M. R. Peres, K. S. Novoselov, and A. K. Geim, "The electronic properties of graphene," Reviews of Modern Physics, vol. 81, no. 1, pp. 109-162, 2009.

[3] C. L. Kane and E. J. Mele, "Quantum spin hall effect in graphene," Physical Review Letters, vol. 95, no. 22, Article ID 226801, pp. 1-4, 2005.

[4] H. Min, J. E. Hill, N. A. Sinitsyn, B. R. Sahu, L. Kleinman, and A. H. MacDonald, "Intrinsic and Rashba spin-orbit interactions in graphene sheets," Physical Review B, vol. 74, no. 16, Article ID 165310, 5 pages, 2006.

[5] D. Huertas-Hernando, F. Guinea, and A. Brataas, "Spinorbit coupling in curved graphene, fullerenes, nanotubes, and nanotube caps," Physical Review B, vol. 74, no. 15, Article ID 155426, 15 pages, 2006.

[6] Y. Yao, F. Ye, X.-L. Qi, S.-C. Zhang, and Z. Fang, "Spin-orbit gap of graphene: first-principles calculations," Physical Review $B$, vol. 75, no. 4, Article ID 041401, 4 pages, 2007.

[7] O. Seiichiro, I. Yasuhito, K. Hiroshi, and J.-I. Inoue, "Intrinsic spin hall effect in graphene: numerical calculations in a multiorbital model," Physical Review B, vol. 78, no. 12, Article ID 121403, 4 pages, 2008.

[8] M. Gmitra, S. Konschuh, C. Ertler, C. Ambrosch-Draxl, and J. Fabian, "Band-structure topologies of graphene: spin-orbit coupling effects from first principles," Physical Review B, vol. 80, no. 23, Article ID 235431, 5 pages, 2009.

[9] A. De Martino, R. Egger, K. Hallberg, and C. A. Balseiro, "Spin-orbit coupling and electron spin resonance theory for carbon nanotubes," Physical Review Letters, vol. 88, no. 20, Article ID 206402, pp. 1-4, 2002.

[10] F. Kuemmeth, S. Ilani, D. C. Ralph, and P. L. McEuen, "Coupling of spin and orbital motion of electrons in carbon nanotubes," Nature, vol. 452, no. 7186, pp. 448-452, 2008.

[11] A. H. Castro Neto and F. Guinea, "Impurity-induced spinorbit coupling in graphene," Physical Review Letters, vol. 103, no. 2, Article ID 026804, 4 pages, 2009.

[12] K. Nakada, M. Fujita, G. Dresselhaus, and M. S. Dresselhaus, "Edge state in graphene ribbons: nanometer size effect and edge shape dependence," Physical Review B, vol. 54, no. 24, pp. 17954-17961, 1996.
[13] L. Brey and H. A. Fertig, "Edge states and the quantized Hall effect in graphene," Physical Review B, vol. 73, no. 19, Article ID 195408, 5 pages, 2006.

[14] L. Brey and H. A. Fertig, "Electronic states of graphene nanoribbons studied with the Dirac equation," Physical Review $B$, vol. 73, no. 23, Article ID 235411, 5 pages, 2006.

[15] Y. Kobayashi, K. Fukui, T. Enoki et al., "Edge state on hydrogen-terminated graphite edges investigated by scanning tunneling microscopy," Physical Review B, vol. 73, Article ID 125415, 8 pages, 2006.

[16] Y. W. Son, M. L. Cohen, and S. G. Louie, "Energy gaps in Graphene nanoribbons," Physical Review Letters, vol. 97, Article ID 216803, 4 pages, 2006.

[17] S. Okada, "Energetics of nanoscale graphene ribbons: edge geometries and electronic structures," Physical Review B, vol. 77, no. 4, Article ID 041408, 4 pages, 2008.

[18] Y. W. Son, M. L. Cohen, and S. G. Louie, "Half-metallic graphene nanoribbons," Nature, vol. 444, no. 7117, pp. 347349, 2006.

[19] L. Yang, C.-H. Park, Y.-W. Son, M. L. Cohen, and S. G. Louie, "Quasiparticle energies and band gaps in graphene nanoribbons," Physical Review Letters, vol. 99, no. 18, Article ID 186801, 4 pages, 2007.

[20] M. Zarea and N. Sandler, "Electron-electron and Spin-orbit interactions in armchair graphene ribbons," Physical Review Letters, vol. 99, no. 25, Article ID 256804, 4 pages, 2007.

[21] M. Zarea, C. Büsser, and N. Sandler, "Unscreened coulomb interactions and the quantum spin hall phase in neutral zigzag graphene ribbons," Physical Review Letters, vol. 101, no. 19, Article ID 196804, 4 pages, 2008.

[22] M. Zarea and N. Sandler, "Quantum spin hall phase in neutral zigzag graphene ribbons," Physica B, vol. 404, no. 18, pp. 26942698, 2009.

[23] G. Kresse and J. Furthmüller, "Efficient iterative schemes for ab initio total-energy calculations using a plane-wave basis set," Physical Review B, vol. 54, no. 16, pp. 11169-11186, 1996.

[24] P. E. Bläuohl, "Projector augmented-wave method," Physical Review B, vol. 50, pp. 17953-17979, 1994.

[25] J. P. Perdew, K. Burke, and M. Ernzerhof, "Generalized gradient approximation made simple," Physical Review Letters, vol. 77, no. 18, pp. 3865-3868, 1996.

[26] M. P. Lopez-Sancho and M. C. Munoz, "Intrinsic spinorbit interactions in flat and curved graphene nanoribbons," Physical Review B, vol. 83, no. 7, Article ID 075406, 9 pages, 2011. 

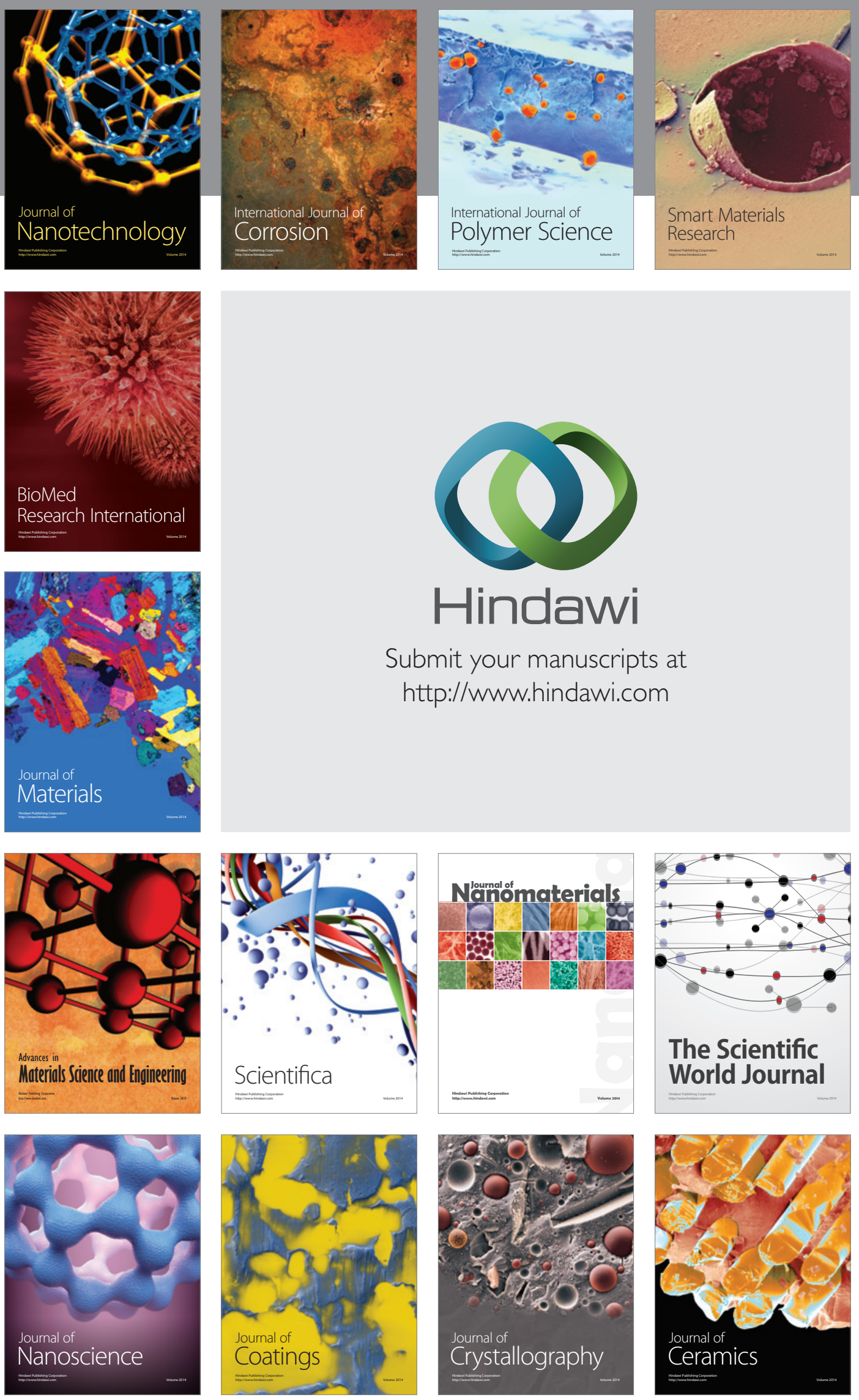

The Scientific World Journal

Submit your manuscripts at

http://www.hindawi.com

\section{World Journal}

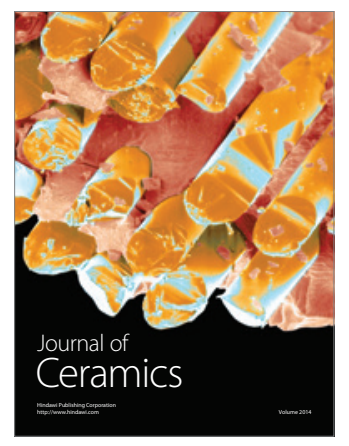

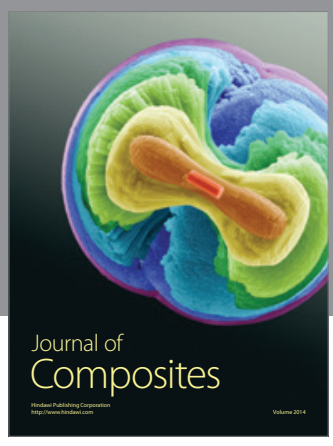
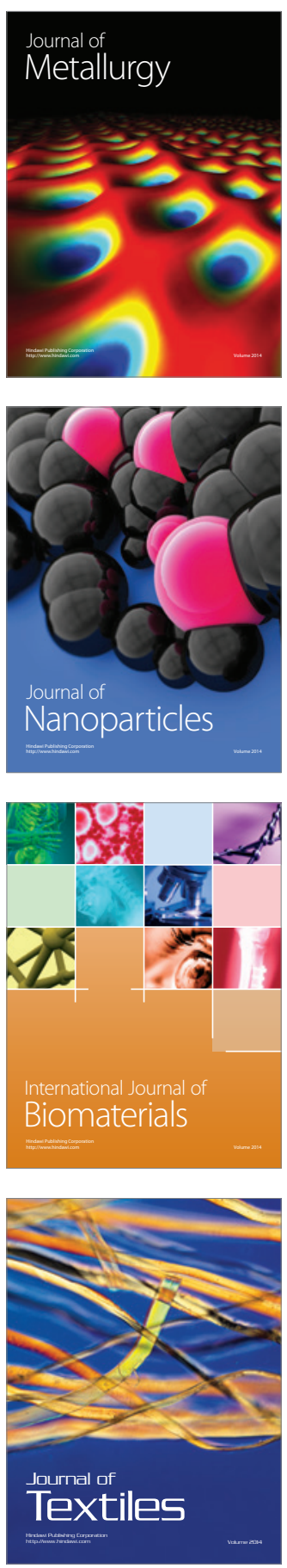